\title{
La réception précoce de la pensée économique autrichienne en Europe centrale
}

Die frühe Rezeption des österreichischen Wirtschaftsdenkens in Mitteleuropa

The early reception of Austrian economic thought in Central Europe

Julius Horváth et Tomáš Krištofóry

\section{CpenEdition}

\section{Journals}

Édition électronique

URL : https://journals.openedition.org/austriaca/1022

DOI : 10.4000/austriaca.1022

ISSN : 2729-0603

Éditeur

Presses universitaires de Rouen et du Havre

Édition imprimée

Date de publication : 1 juin 2020

Pagination : 49-65

ISBN : 979-10-240-1492-0

ISSN : 0396-4590

\section{Référence électronique}

Julius Horváth et Tomáš Krištofóry, «La réception précoce de la pensée économique autrichienne en Europe centrale », Austriaca [En ligne], 90 | 2020, mis en ligne le 01 juin 2021, consulté le 29 septembre 2022. URL : http://journals.openedition.org/austriaca/1022 ; DOI : https://doi.org/10.4000/austriaca. 1022

Ce document a été généré automatiquement le 29 septembre 2022.

Tous droits réservés 


\section{La réception précoce de la pensée économique autrichienne en Europe centrale}

Die frühe Rezeption des österreichischen Wirtschaftsdenkens in Mitteleuropa

The early reception of Austrian economic thought in Central Europe

Julius Horváth et Tomáš Krištofóry

\section{Introduction}

1 L'école autrichienne d'économie doit sa naissance aux travaux de Carl Menger (1840-1921) à Vienne. À partir du début des années 1880, un groupe de disciples de Menger développe davantage ses concepts, dans plusieurs sites et selon plusieurs directions.

Dans l'entrée «École autrichienne " d'une des premières éditions du Palgrave's Dictionary of Economics, Friedrich von Wieser lui-même écrivait que l'école autrichienne avait commencé par la publication des Grundsätze der Volkswirthschaftslehre de Menger en 1871, l'ouvrage devenu célèbre que complétèrent les travaux d'Eugen von BöhmBawerk (et les siens propres) ${ }^{1}$. Avant 1900, quelques-uns des disciples de Menger, dont les deux susnommés, obtiennent des postes universitaires importants au sein d'universités de la monarchie des Habsbourg' ${ }^{2}$, avant de prendre des positions politiques majeures - ils furent plus tard tous les deux ministres impériaux.

3 C'est dans les universités germanophones de l'Empire autrichien que les idées de l'école autrichienne commencèrent ainsi à se répandre. Après la première guerre mondiale, quelques-unes de ces universités allaient appartenir aux États nouvellement indépendants d'Europe centrale, à savoir la Tchécoslovaquie, la Pologne et la Hongrie, ainsi qu'au sud, la Yougoslavie. La pensée autrichienne exerce ainsi son influence d'abord dans les territoires qui se trouvent au sein des frontières de l'empire austro- 
hongrois et, plus tard, ces idées continuent leur expansion à travers les frontières des États-nations nouvellement créés.

4 Ce n'est pas là une réception passive, mais un processus actif, car on peut observer que certains économistes d'Europe centrale et de souche non autrichienne ont aussi contribué au développement de la pensée économique dite "autrichienne». Des économistes autrichiens éminents ont rappelé la diffusion de ces idées en Europe centrale ainsi que la contribution de ces économistes au développement de cette école de pensée autrichienne. Par exemple, Friedrich Hayek écrit qu'immédiatement après l'émergence de l'école autrichienne (soit, vers le milieu des années 1880), les idées de l'école se sont propagées au sein d'autres nations de la monarchie: "Au cours des années suivantes, de nombreux adeptes apparaissent parmi les économistes tchèques, polonais et hongrois de la monarchie austro-hongroise ${ }^{3} »$.

5 Ludwig von Mises devait quant à lui souligner l'importance de la contribution des contributeurs d'Europe centrale :

Parmi les disciples de Menger, de Böhm-Bawerk et de Wieser, il y avait également des Autrichiens non allemands. Deux disciples tchèques se sont particulièrement distingués par leur contribution éminente : Franz Čuhel et Karel Engliš ${ }^{4}$.

Cet article étudie l'influence des débuts de la pensée économique autrichienne en Europe centrale. Comme, au cours de son élaboration, le sujet s'est révélé beaucoup plus complexe qu'il ne nous paraissait d'abord, à nous auteurs, nous ne pourrons présenter, dans le cadre d'un texte aussi court, qu'une petite partie de sa richesse et de sa complexité. Ainsi, nous nous limitons sciemment à l'avant-seconde guerre mondiale, sans pour autant pouvoir développer la polémique majeure autour de la rationalité du calcul (des prix et des productions) sous le régime socialiste. Nous n'allons pas non plus nous engager dans l'interprétation textuelle.

7 Après des rappels sur les circuits suivis par la diffusion de la pensée économique en Europe centrale dans l'introduction, nous tenterons d'évaluer, dans la deuxième partie, l'impact général de l'école autrichienne à ses débuts sur l'évolution ultérieure de la pensée économique en Europe centrale, en ce qui concerne la période précédant la première guerre mondiale et la période de l'entre-deux-guerres. Nous apporterons au lecteur des éclaircissements sur le contexte social, et nous indiquerons le rôle de quelques économistes majeurs. Dans la troisième partie, nous esquisserons la manière dont les économistes appartenant à diverses souches ethniques d'Europe centrale purent contribuer à forger la pensée économique " autrichienne ».

\section{Diffusion de la pensée autrichienne en Europe centrale}

8 Les pays indépendants d'Europe centrale sont nés de la première guerre mondiale. Cependant, bien avant elle, dans les grandes villes comme Budapest, Prague, Brno, Ljubljana, Cracovie, Lvov, Bratislava et Varsovie, on pouvait observer un essor intellectuel qui allait se poursuivre tout au long de l'entre-deux-guerres. C'est alors qu'on put observer l'institutionnalisation et la professionnalisation de l'économie en tant que discipline universitaire dans ces pays. L'amélioration de la qualité de ces universités et de leur production scientifique est considérée comme faisant partie intégrante de la renaissance nationale des pays en question ${ }^{5}$. 
9 Avant la première guerre mondiale, au sein de ces nations d'Europe centrale, l'Autriche est considérée non seulement comme le pays le plus développé, celui qui a les meilleures universités de la région, mais aussi comme celui qui constitue l'oppresseur des ambitions nationales. En d'autres termes, l'acceptation de la pensée économique autrichienne se mêlait à l'impact des vues politiques de et sur l'Autriche. Les leaders nationalistes d'Europe centrale adoptent alors des attitudes variées vis-à-vis de l'Autriche : parfois, ils préfèrent la révolte à son encontre, parfois une coopération plus poussée. On peut observer de telles différences dans les avis et dans les comportements également chez les économistes d'Europe centrale ${ }^{6}$.

10 Jusqu'en 1918, l'éducation des membres des groupes ethniques d'Europe centrale dans le domaine économique avait évidemment été assurée au sein des institutions des grands empires, et donc principalement en langue allemande ou hongroise. La diffusion internationale de la pensée économique autrichienne était facilitée par le fait qu'elle rencontrait très peu de barrières linguistiques, car les classes éduquées maîtrisaient couramment ces langues (au moins l'allemand). Néanmoins, avant la première guerre mondiale, les universités de langue allemande ou hongroise rendirent plus difficile l'obtention du poste de professeur universitaire aux économistes tchèques, polonais, slovaques ou slovènes ayant ouvertement une orientation «nationale ». Krameš mentionne le cas de František Ladislav Rieger (ainsi que celui de Maximilian Wellner), dans les années 1860 à Prague 7 .

11 La situation allait changer, dans une certaine mesure, après la création de l'université bilingue Karl-Ferdinand en $1882^{8}$. Ce furent surtout les étudiants autochtones, enhardis par les mouvements de renaissance nationale, qui allaient exiger que leur langue maternelle devînt langue d'enseignement universitaire. En 1882, leurs forces unies amenèrent le gouvernement autrichien à établir l'université de Prague en deux entités parallèles : une partie tchèque et une partie allemande. Comme l'université allemande de Prague prenait de l'importance avec l'avènement de l'école autrichienne dans les années 1880 , cela contribuait à améliorer par ricochet la qualité de l'enseignement de la partie tchèque de l'université.

12 En Europe centrale, le grand public était surtout informé des différentes écoles de pensée par le biais de manuels étrangers de vulgarisation portant sur l'histoire de la pensée économique. Le plus populaire d'entre eux était sans doute celui rédigé par deux auteurs français, Charles Gide et Charles Rist. À cette époque-là, cet ouvrage s'impose comme le manuel d'histoire de la pensée économique qui fait autorité en Europe centrale. Jan Koudela, un disciple d'Albín Bráf, le traduit en tchèque avec Milada Koudelová dans les premières années $\mathrm{du} \mathrm{xx}^{\mathrm{e}}$ siècle ${ }^{9}$. Wlodzimierz Czerkawski le traduit en polonais à peu près à la même époque ${ }^{10}$. Ces traductions $d u$ manuel français présentent d'excellentes introductions à la pensée autrichienne.

13 À partir des années 1880 , le niveau de la pensée économique dans les langues tchèque, hongroise et polonaise s'améliore peu à peu par rapport aux décennies précédentes. La présence de professeurs autrichiens et les contacts avec ces derniers contribuent fondamentalement au développement de la pensée économique en Europe centrale au sein des communautés autochtones. Par exemple, le développement de la pensée tchèque bénéficie largement du fait que Friedrich von Wieser enseigne à Prague, et qu'Albín Bráf, qui allait préparer une génération entière d'éminents économistes tchèques, avait été en contact étroit avec Carl Menger ${ }^{11}$. L'influence de Wieser fut prédominante, comme les mémoires de Hayek et de Morgenstern en témoignent à leur 
tour $^{12}$. En Pologne, entre les années 1890 et 1910, c'est Wlodimierz Czerkawski qui joue un rôle similaire à celui de Bráf dans le réveil national tchèque ${ }^{13}$. Tous les deux deviennent des personnages clés pour l'implantation de la pensée économique autrichienne dans le monde académique d'Europe centrale.

Rappelons d'ailleurs ici que le fondateur de l'école dite « autrichienne », Carl Menger, avait fait ses études à l'université allemande de Prague, puis à l'université Jagellon de Cracovie, au début des années 1860. Les chaires d'économie de l'université allemande de Prague, de l'Institut allemand de technologie de Prague et de l'Institut allemand de technologie de Brno furent occupées après qu'il eût fondé son école, soit par les membres de cette école autrichienne d'économie, soit par des représentants de l'école historique allemande, et ce de façon continue entre 1879 et le milieu des années 1930. Le débat entre les écoles trouvait là une place importante. La liste des enseignants de Prague de cette époque est particulièrement impressionnante ${ }^{14}$.

15 Jusqu'en 1918, la Pologne est restée partagée entre la Prusse, la Russie et l'Autriche. La sphère d'influence autrichienne joua un rôle particulièrement important dans le développement de la pensée économique polonaise, en raison de sa liberté académique relativement plus grande qui régnait dans l'empire austro-hongrois. Autour des années 1870, l'université de Cracovie et celle de Lvov deviennent plus "polonaises" dans l'âme, disons, en particulier Cracovie qui se transforme en un centre universitaire important. Le mélange très particulier des idées venant de la division en trois parties de la Pologne ouvre la voie à des contributions importantes grâce au brassage de ces traditions sous l'effet des influences venues par ailleurs d'Occident. Dans les années 1860, Menger étudie à Cracovie avec Julian Dunajewski, ancien ministre autrichien des Finances ${ }^{15}$. Il est ensuite, pendant un certain temps, journaliste économique pour la gazette locale à Lvov (alors connue sous le nom de Lemberg).

$16 \mathrm{Au} \mathrm{xx} x^{\mathrm{e}}$ siècle, les contributions majeures comprennent notamment, en 1922, le livre de Roman Rybarski intitulé Wartość, kapitał i dochód (Valeur, capital et revenu) qui s'occupe de la théorie du prix de Böhm-Bawerk ${ }^{16}$, puis l'apport éminent d'Oskar Lange au débat sur la question du calcul économique en régime socialiste. La lutte avec les économistes autrichiens, en particulier Mises, constitue un des grands débats des années $1930^{17}$.

En Hongrie, la situation différait quelque peu en raison de la place obtenue dans l'empire habsbourgeois par le compromis de 1867. En ce qui concerne la pensée économique, les économistes hongrois de l'époque les plus influents furent Gyula Kautz, Ákos Navratil et Béla Földes : ils penchaient tous plutôt pour l'école historique allemande ${ }^{18}$. Selon l'historien hongrois Zsuzsa Bekker, ces éminents économistes étaient en fait à mi-chemin entre ces différentes écoles de pensée, à la croisée des chemins pour ainsi dire et ce furent leurs héritiers qui prirent des positions plus arrêtées (mais pas toujours heureuses) ${ }^{19}$. Theo Surányi-Unger adopte un courant organiciste de l'influence autrichienne en devenant adepte de Spann ${ }^{20}$. Cependant, du fait de sa position particulière privilégiée de 1867 à la disparition de l'empire des Habsbourg, la Hongrie a surtout produit des penseurs qui déménageaient régulièrement vers l'Autriche, ou dont les parents s'y étaient déjà établis, de sorte qu'ils y étaient nés et contribuaient d'emblée à l'essor de la pensée autrichienne, et principalement viennoise. Parmi ceux-ci, on trouve Eugen Peter Schwiedland et Julius Friedrich Gans von Ludassy ${ }^{21}$, qui étaient directement membres de l'école autrichienne. Cependant, le représentant le plus important de la pensée autrichienne en Hongrie est 
Farkas Heller qui reste ouvert aux vues de l'école historique ${ }^{22}$; en quelque sorte, Heller essaie de faire la synthèse des deux écoles ${ }^{23}$.

Le cas slovaque est un peu différent. Jusqu'en 1919, la Slovaquie n'avait pas d'université enseignant dans sa propre langue. Le développement de la pensée économique y connut donc un retard certain, non seulement par rapport à l'Autriche, mais aussi par rapport aux autres nations d'Europe centrale. La proximité avec Vienne, de l'autre côté du Danube, était à cet égard le facteur explicatif majeur. En 1919, l'université Comenius fut toutefois fondée à Bratislava. Le nombre des Slovaques aptes à occuper des postes de professeur dans la nouvelle université étant faible, les premiers professeurs d'économie nommés par le ministère de Prague étaient d'origine tchèque ${ }^{24}$. Ce sont eux qui communiquèrent paradoxalement l'influence autrichienne.

En 1922, Antonín Basch, tchèque de naissance, rejoint la nouvelle faculté. Basch a fait ses études à Vienne. Il y suit des cours et des conférences, entre autres, par Max Weber. Basch est un professeur important à l'université Comenius, et il expose à ses étudiants un certain nombre d'idées de l'école autrichienne dès le début ${ }^{25}$. Cet intérêt, une fois stimulé, ne devait plus cesser chez nombre d'étudiants de cette universitée ${ }^{26}$.

Avec des décennies de retard, les premières contributions nettement slovaques en économie attendent d'être publiées dans les années 1930 et 1940, surtout grâce à l'activité d'Imrich Karvaš et de Rudolf Briška. Dans son manuel, de presque sept cents pages, Briška consacre des passages essentiels à la pensée de l'école autrichienne qu'il appelle l'« école des bénéfices marginaux ${ }^{27}$ " Dans son manuel écrit avant la seconde guerre mondiale, Karvaš, quant à lui, n'ignore pas non plus les contributions des Autrichiens. Bien au contraire, il décrit systématiquement et longuement la contribution de l'école autrichienne ${ }^{28}$.

21 Évoquons pour conclure ce passage en revue des pays d'Europe centrale nés de l'éclatement de l'empire habsbourgeois, le cas de la Slovénie. Dès le début des années 1900, des universitaires slovènes avaient obtenu des bourses d'études pour des universités autrichiennes, dans la perspective de devenir ensuite des professeurs de la nouvelle université de Ljubljana ${ }^{29}$. Quand une université entre effectivement en activité à Ljubljana en 1919, la proportion de professeurs slovènes est clairement plus importante que le pourcentage des enseignants slovaques à l'université Comenius, par exemple. Un manuel d'économie en langue slovène, publié par Valentin Žun dès 1908, contribue à structurer la terminologie et il constitue le point de départ du développement d'une pensée économique en Slovénie et en langue slovène alors qu'à titre de comparaison, la terminologie économique en langue slovaque se met au point dans les années $1930^{30}$. L'historien Aleksander Bilimovič a considéré que le manuel de Žun avait exercé une influence déterminante sur le développement de la pensée économique slovène ${ }^{31}$.

Il convient enfin de mentionner le rôle que Ivan Žmavc et Milko Brezigar jouèrent aux débuts de la pensée économique slovène ${ }^{32}$. Néanmoins, en 1920 , le département d'économie politique de Ljubljana allait être dirigé par un économiste plus connu, dont le nom reste associé à la Slovénie de l'entre-deux-guerres, Aleksander D. Bilimovič. Né en Ukraine, il a étudié en Allemagne, fui l'Union soviétique où il était rentré et, après son installation à Ljubljana, il devait publier régulièrement ses travaux dans des ouvrages allemands et autrichiens. Souvent invité à Vienne pour donner des cours et des conférences, Bilimović reste à Ljubljana jusqu'en 1945, date à laquelle il doit fuir aux États-Unis. Bilimović a entretenu une correspondance nourrie avec certains 
représentants majeurs de l'école autrichienne ${ }^{33}$. Malgré des options parfois bien peu libérales, Bilimović restait proche de la pensée autrichienne et il polémiqua en langue allemande avec Karel Engliš eu égard à leurs systèmes respectifs en matière d'économie ${ }^{34}$.

\section{Contribution des économistes d'Europe centrale à la pensée autrichienne}

Nous concentrons notre attention dans la partie qui suit sur quelques auteurs tchèques $^{35}$. Dans son livre publié en 1907, Franz Čuhel étudie la théorie des besoins de l'agent économique en adoptant un point de vue explicitement psychologique. Il est de fait que Krameš mentionne que Menger avait recommandé le livre à Böhm-Bawerk, qui en a écrit une excellente recension pour la maison d'édition d'Innsbruck ${ }^{36}$.

Selon Marek Hudík ${ }^{37}$, la théorie ordinale des besoins que Čuhel proposait en insistant sur l'impossibilité de toute comparaison interpersonnelle d'utilité constitue le fondement de la microéconomie moderne. C'est sans doute extrapoler l'importance du débat entre ordinalité et cardinalité, mais il est de fait que le livre de Čuhel est souvent cité, notamment par Eugen Slutsky ${ }^{38}$, Wesley Clair Mitchell ${ }^{39}$, Theo Surányi-Unger ${ }^{40}$, et Fritz Machlup ${ }^{41}$. En explorant les archives, nous avons retrouvé une dissertation inédite de Hayek sur la question de l'imputation des facteurs qui date de 1923, où il cite aussi Čuhel, qui peut donc apparaître comme une de ses sources d'inspiration ${ }^{42}$. Des examens plus critiques ont indiqué les formulations maladroites dans l'ouvrage de l'économiste tchèque et les positions sont partagées sur le rôle de la psychologie au regard de la difficulté de créer une passerelle entre la psychologie et l'économie dans l'école autrichienne ${ }^{43}$.

Il est possible de prouver encore que l'ouvrage de Čuhel a eu un impact sur l'analyse de l'utilité marginale par Mises, qui incorpore les idées de Čuhel pour souligner que l'utilité marginale ne peut pas être mesurable en tant que quantité mathématique, c'est-à-dire que le chercheur ne peut jamais se prévaloir de calculer une utilité totale comme étant la somme des utilités marginales: "[la théorie qui veut] que la valeur subjective ne soit pas mesurable et ne puisse par conséquent pas être calculée, fut donc prouvée pour la première fois par Franz Čuhel ", n'hésitent ainsi pas à écrire des historiens misésiens ${ }^{44}$. Cependant, l'étoile de Čuhel devait commencer à pâlir assez vite, principalement en raison de ses propres problèmes de santé.

L'autre figure que nous souhaitons évoquer ici est celle de Karel Englišs ${ }^{45}$. Il participa très activement à la fois à la vie politique et universitaire. Il fut élu au Parlement tchécoslovaque et il fut l'un des fondateurs de l'université Masaryk de Brno, dont il assuma le premier le poste de recteur. Il était probablement le plus proche de la vision que Bráf avait donnée pour ce qui est de l'émergence d'un système d'économie tchèque bien distinct ${ }^{46}$. La téléologie d'Engliš a des traits communs avec certains aspects de la praxéologie que Mises a mis en avant. Les deux penseurs se connaissaient et se rendaient visite ${ }^{47}$. Kirzner fit à son tour l'éloge d'Engliš et compara son approche d'Engliš à la praxéologie de Mises ${ }^{48}$.

Mises ne précise cependant nulle part à notre connaissance ce qu'il considérait luimême devoir être la contribution d'Engliš aux conceptions de l'école autrichienne, même s'il faisait l'éloge de sa contribution comme membre. Sans recherche 
approfondie dans les archives, on ne peut que supposer ce que Mises en pensait vraiment. Le fait est qu'Engliš avait délaissé la sociologie au milieu des années 1910 et il est possible que ce choix ait influencé Mises dans son propre cheminement identique un peu plus d'une décennie plus tard ${ }^{49}$.

Entre autres ouvrages où Mises mentionne Englišs, L'action humaine n'est pas le moindre et l'auteur tchèque y apparaît lorsque Mises débat de la causalité et de la téléologie comme étant les deux seules approches disponibles à une approche cohérente de la science: "Le changement peut être conçu comme le résultat de l'opération de la causalité mécanique ou du comportement intentionnel; il n'y a pas de troisième voie pour l'esprit humain ${ }^{50}$.»

L'argumentation de Hayek fait à son tour voir que les Autrichiens regardèrent la contribution d'Engliš sur la téléologie comme caractéristique de sa position méthodologique. La téléologie, telle celle prônée par Engliš, ne peut pas être considérée comme l'approche adéquate pour la science et ce qu'elle apporte de normatif n'est pas scientifique. Toutefois, l'économie n'est pas toujours une science positive, du moins selon Engliš. Alors que, pour l'école autrichienne, l'économie est avant tout une science positive de l'action humaine, Engliš s'en démarque donc en quelque manière en indiquant que, pour lui, les sciences économiques ne sont ni positives ni normatives, mais qu'elles forment un type de science bien distinct, une science téléologique. L'idée lui est propre et il conviendra à ses exégètes d'explorer plus avant les possibilités de la développer.

En guise de conclusion, indiquons seulement pour terminer cette brève introduction que notre article visait à présenter les pistes pour une analyse de l'influence intellectuelle de l'école autrichienne d'économie dans le milieu intellectuel des pays d'Europe centrale jusqu'à la seconde guerre mondiale. Nous avons montré que cette influence était réciproque entre Vienne et les pays qui avaient connu sa domination et qui prirent leur indépendance à la chute de l'empire des Habsbourg. Elle était disproportionnée, de toute évidence, mais effectivement mutuelle depuis les années 1880. Il est donc possible de parler d'un processus d'importation des idées de l'école autrichienne dans les communautés économiques naissantes chez les populations non allemandes d'Europe centrale. Le transfert des idées autrichiennes fut le plus prononcé vers les régions de langue tchèque, dont les ressortissants contribuèrent également, dans une certaine mesure, au progrès des connaissances de l'école autrichienne, toutes les nations d'Europe centrale étant dans une certaine mesure fortement influencées par l'émergence de cette école de pensée économique alors toute nouvelle.

\section{BIBLIOGRAPHIE}

N. B. Les titres ci-dessous s'ajoutent aux références figurant dans les notes, afin de constituer une bibliographie sur l'école autrichienne des origines dans les pays d'Europe centrale. 
BASCH Antonin, « Review of Omnipotent Government by Ludwig Von Mises », The American Economic Review, vol. 34, $\mathrm{n}^{\circ}$ 4, décembre 1944, p. 899-903.

CUHEL Franz, Zur Lehre von den Bedürfnissen. Theoretische Untersuchungen über das Grenzgebiet von Ökonomik und Psychologie, Innsbruck, Wagner, 1907.

DOLEŽALOVÁ Antonie, Střední Evropa na cestě od minulosti k budoucnosti (L'Europe centrale sur le chemin du passé vers l'avenir), Prague, Centrum středoevropských studií, 2014.

ENGLIŠ Karel, « Tschechoslowakei », dans Hans Mayer (éd.), Die Wirsthaftstheorie der Gegenwart, Wien, Julius Springer, 1927, vol. 1.

ENGLIŠ Karel, Der relative Nutzen und der Grenznutzen: zur Grenznutzenlehre, Wien, [s. n.], 1928.

ENGLIŠ Karel, Soustava národního hospodářství (Système de l'économie nationale), Praha, Melantrich, 1938.

HÁLEK Jan, « Ivan Žmavc (1871-1956) », Akademický Bulletin, oficiální časopis Akademie věd ČR, $\mathrm{n}^{\circ} 12$, 2006, http://abicko.avcr.cz/archiv/2006/12/09/ (consulté le 16 septembre 2020).

HAYEK Friedrich August (dir.), Beiträge zur Geldtheorie, Wien, Springer, 1933.

HELLER Farkas, A közgazdasági elmélet története (Histoire de la théorie économique), Pécs, Dunántúl Pécsi Egyetemi Könyvkiadó és nyomda, 1945.

HIGGS Henry, « Friedrich von Wieser, 1851-1926 », Economica, n² 20, juin 1927, p. 150-154.

KLAUSINGER Hansjörg, « Krise und Niedergang der Nationalökonomie an der Wiener Universität nach 1917 », Schriften des Vereins für Socialpolitik, vol. 115, 2016.

KRAMEŠ Jaroslav, Ekonomie jako předmět výuky na vysokých školách v českých zemích do roku 1918 (Les sciences économiques comme matière au programme d'études universitaires dans les pays tchèques jusqu'en 1918), Praha, Typograf, 2012.

KRAMEŠ Jaroslav, « Professor Albín Bráf, his Students and the Austrian School », Prague, VŠE, 2016, https://msed.vse.cz/msed_2016/article/70-Krames-Jaroslav-paper.pdf.

LANGE Oskar, Pisma ekonomiczne i społeczne 1930-1960 (Écrits économiques et sociaux, 1930-1960), Warszawa, Państwowe Wydawnictwo Naukowe, 1961.

MÁTYÁs Antal, « Adalék Heller Farkas elméleti munkásságához » (Contribution sur le travail théorique de Farkas Heller), Közgazdasági Szemle, vol. 45, 1998, p. 738-746.

MÁTYÁs Antal, « 175 éve született a korszerű magyar közgazdaságtan első nagy képviselője, Kautz

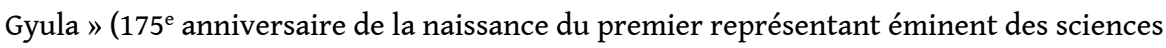
économiques modernes en Hongrie, Gyula Kautz), Közgazdasági Szemle, vol. 52, 2005, p. 613-622.

MAYER Hans (dir.), Die Wirtschaftstheorie der Gegenwart, Wien, Julius Springer, 1927.

MAYER Hans, Human Action, Auburn, Ludwig von Mises Institute, 2004.

MAYER Hans et SPIETHOFF Arthur (éd.), Probleme der Wertlehre, München, Duncker \& Humblot, 1931-1933, 2 vol.

PHILIPPOVICH Eugen von, Die Entwicklung der wirtschaftspolitischen Ideen im 19. Jahrhundert, Tübingen, Mohr, 1910 (trad. tchèque : Vývoj hospodářsko-politických idejí v XIX. Století, přel. J. B. Novák, Praha, R. Brož, 1911]. ROSENSTEIN-RODAN Paul N., «The Role of Time in Economic Theory », Economica, vol. 1, n 1, 1934, p. 77-97. 


\section{NOTES}

1. Henry Higgs, « Friedrich von Wieser, 1851-1926 », Economica, nº 20, juin 1927, p. 150.

2. Par exemple, entre 1880 et 1889 , Böhm-Bawerk occupe un poste universitaire à Innsbruck. Entre 1884 et 1903, Wieser a un poste universitaire à Prague, Robert Zuckerkandl de même, de 1894 à 1926, et Emil Sax, de 1879 à 1893.

3. Friedrich August Hayek, The Fortunes of Liberalism, London, Routledge, 1992, p. 83 : "In the following years numerous adherents also appeared among the Czech, Polish, and Hungarian economists of the Austro-Hungarian monarchy. »

4. Ludwig von Mises, Notes and Recollections, Indianapolis, Liberty Fund, 2013, p. 151.

5. À titre d'exemple, l'historien Jaroslav Krameš mentionne Albín Bráf, un des premiers économistes savants à écrire en langue tchèque, qui voyait la renaissance économique comme une étape dans la renaissance nationale, et la création d'une école de pensée tchèque comme la phase ultime de cette renaissance économique : Jaroslav Krameš, Profesor Albín Bráf a jeho žáci: česká národohospodářská škola Bráfova (Le professeur Albín Bráf et ses disciples: l'École d'économie nationale de Bráf), Praha, Národohospodářský ústav Josefa Hlávky, 2016, p. 21-35. Bráf avait débuté sa carrière sous l'influence de la première École historique allemande. Plus tard, il se rapprocha des Autrichiens à l'occasion du Methodenstreit.

6. Par exemple, l'historien Jerzy Chodorowski indique que Roman Rybarski, économiste polonais qui, parti de Cracovie, avait étudié au Royaume-Uni, en France, en Italie et aux États-Unis, évitait intentionnellement les pays germanophones. Voir Jerzy Chodorowski, Roman Rybarski (1887-1942): $z$ historii polskiej myśli ekonomicznej i prawnoustrojowej pierwszej połowiny xx wieku (Roman Rybarski [1887-1942]: histoire de la pensée économique et politique polonaise de la première moitié du $\mathrm{xx}^{e}$ siècle), Wrocław, Wydawnictwo Uniwersytetu Wrocławskiego, 1997. Inversement, son collègue Adam Heydel participa aux séminaires de Mises à Vienne et travailla à améliorer les relations avec l'Autriche. Des exemples similaires se trouvent dans les autres pays d'Europe centrale: des économistes d'Europe centrale de souche non autrichienne occupaient d'ailleurs de hautes fonctions dans les gouvernements des Habsbourg: citons Josef Kaizl (1854-1901), Albín Bráf (1851-1912), Julian Dunajewski (1821-1907), Leon von Bilinski (1846-1923) et Gyula Kautz (1829-1909), qui servirent tous en tant que ministres, gouverneurs ou hauts-fonctionnaires de l'Empire.

7. Rieger, journaliste et écrivain éminent, militait pour le libre-échange. Il reçut le surnom de «Bastiat tchèque » en référence au libéral français, Frédéric Bastiat. Wellner est moins connu. Voir Jaroslav Krameš, op. cit.

8. Jan Havránek (dir.), Dějiny Univerzity Karlovy III (1802-1918) (Histoire de l'université Charles-III, 1802-1918), Praha, Karolinum, 1997.

9. Charles Gide et Charles Rist, Dějiny nauk národohospodářských od doby fysiokratů až po naše dny (Histoire des doctrines économiques, depuis les physiocrates jusqu'à nos jours), Praha, Jan Laitcher, 1915-1917, 2 vol. La seconde édition tchèque parut en 1928 dans Prague indépendante.

10. Jerzy Chodorowski, op. cit., 1997, p. 53.

11. Jaroslav Krameš, op. cit.

12. Hayek narre que les étudiants de Wieser ne devaient jamais oublier sa personnalité impressionnante. Ses propos sont rapportés par Higgs, op.cit., p.152. En outre, Oskar Morgenstern raconte : «Wieser est vraiment une personnalité remarquable et impressionnante pour tout un chacun ; de par sa personnalité, il exerce une influence déterminante sur ses amis et ses disciples. Sa grande culture, ses intérêts universels, la position qu'il occupe dans la science, la politique et la vie sociale, son urbanité admirable d'aristocrate, qu'il incarne par excellence, voilà ce qui le rend un représentant idéal du vieil Empire austro-hongrois ; entre tous, c'est lui qui a perdu son milieu avec la dissolution de la Monarchie pour devenir un personnage historique » 
(«Friedrich von Wieser, 1851-1926», American Economic Review, vol. 17, n 4, décembre 1927, p. 674).

13. Pour Chodorowski, « c'est grâce à lui [Czerkawski], dans ses deux ouvrages de théorie, que la pensée économique polonaise a pu rattraper la pensée économique occidentale. Depuis les années 1830-1840 (F. Skarbek, L. Wolowski), c'était la première fois que des contributions polonaises en économie satisfaisaient aux normes occidentales " (Jerzy Chodorowski, op. cit., p. 53).

14. Parmi les Autrichiens déjà cités, rappelons les dates des années d'enseignement de Wieser (1884-1903), Sax (1879-1894) et Zuckerkandl (1895-1926). On lit aussi les noms de Hans Mayer (1914-1922), Franz Xaver Weiss (1922-1933), Oskar Engländer (1917-1936) et Hermann von Schullern zu Schrattenhofen (1899-1901) à Brno. La liste comprend des économistes de l'école allemande : Alfred Weber (1904-1908) et Arthur Spiethoff (1909-1917).

15. Outre Dunajewski, l'influence de Peter Mischler est examinée. Peter G. Klein, éditeur de l'ouvrage de Hayek, The Fortunes of Liberalism, conteste la première évaluation de Menger par Hayek où ce dernier affirme que Menger ne fut pas influencé par ces premiers professeurs. Klein cite Erich Streissler qui soutient que Menger fut en effet influencé par un de ses professeurs d'économie à l'université de Prague, Peter Mischler (1821-1864) ; voir Friedrich August Hayek, op. cit., p. 67.

16. Citons de nouveau Heydel qui publie un article en langue allemande dans la revue Wiener Zeitschrift für Nationalökonomie. Heydel narre son séjour à Vienne dans «Sprawodzanie z wyjazdu w celach naukowych za granice w okresie luty-kwiecieńv1928» (Rapport sur mon voyage d'études de février à avril 1928), en ligne (consulté le 16 septembre 2017), http:// kwasnicki.prawo.uni.wroc.pl/pliki/HeydelSprawozdanieWieden1928.pdf. L'historien de la pensée Hansjörg Klausinger a décrit l'activité de Heydel au sein de la Nationalökonomische Gesellschaft de Vienne où Heydel fit connaissance de Rosenstein-Rodan (qui le cite à son tour dans un article de 1934 sur le rôle du temps dans la théorie économique): voir Hansjörg Klausinger, «The Nationalökonomische Gesellschaft in the Interwar Period and Beyond ", Research in the History of Economic Thought and Methodology, vol. 34, 2016, p. 9-43.

17. Paryna mentionne qu'en tant qu'étudiant, Lange écrit une lettre à Heydel, lequel l'aide à poursuivre sa carrière universitaire malgré le fait que les deux adhèrent à des idéologies économiques différentes. Voir Wojciech Paryna, Obrona wolnósci, pokoju, kultury i postepu - Adam Heydel (Défense de la liberté, de la paix, de la culture et du progrès. Adam Heydel), mémoire universitaire, Wroclaw, 2011, p. 24, http://kwasnicki.prawo.uni.wroc.pl/pliki/Paryna_Pracamgr-Adam-Heydel.pdf.

18. Voir Karl von Balás, «Gesamtbild der Forschung in den einzelnen Ländern: Ungarn », dans Hans Mayer (éd.), Die Wirsthaftstheorie der Gegenwart, Wien, Julius Springer, 1927, vol. 1, p. 206. L'ouvrage de Gyula Kautz, Theorie und Geschichte der National-Oekonomik, publié à Vienne en 1858, joua cependant un rôle implicitement important dans le développement du marginalisme. Stanley Jevons mentionne que c'est ce livre qui attira son attention sur l'œuvre de Gossen dans The Theory of Political Economy, $3^{\mathrm{e}}$ éd., London, MacMillan, 1888, p. 15. Gilles Campagnolo a tenté d'évaluer les liens entre Menger et Gossen dans Criticisms of Classical Political Economy: Menger, Austrian Economics and the German Historical School, London, Routledge, 2012, p. 287-301.

19. Zsuzsa Bekker, Szakmai beszámoló, Magyar közgazdasági gondolkodás története c. project (Rapport sur le projet de l'histoire de la pensée économique en Hongrie), 2008, real.mtak.hu/ 826/1/42881_ZJ1.pdf.

20. Selon Othmar Spann, « il y [avait] beaucoup d'économistes qui accept[ai]ent l'idée organiqueuniversaliste d'une manière générale, en émettant toutefois des réserves ». Parmi eux, l'économiste allemand mentionnait notamment Surányi-Unger: voir Othmar Spann, Types of Economic Theory [1929], London, Routledge, 2013, p. 283. 
21. Voir Eugen Maria Schulak et Herbert Unterköfler, The Austrian School of Economics: A History of Its Ideas, Ambassadors, and Institutions, Auburn, Ludwig von Mises Institute, 2011.

22. Antal Mátyás, « Die Österreichische Schule der Nationalökonomie im Spiegel der ungarischen volkswirtschaftlichen Literatur », dans Kurt R. Leube et Andreas Pribersky (dir.), Krise und Exodus. Österreichische Sozialwissenschaften in Mittel Europa, Wien, WUV Universitätsverlag, 1995, p. 74. Heller fait l'éloge du caractère abstrait de l'école autrichienne par rapport à l'école historique. Il saisit aussi le fait que Roscher et Schmoller entendent réfuter la méthode abstraite en se servant de l'argument généralement valide selon lequel la vie économique représente seulement une partie de la vie sociale, plus universelle et qui comporte des caractéristiques ethniques, culturelles, psychologiques, nationales, historiques ainsi que d'autres aspects. Cette objection joue un certain rôle dans ses propres positions.

23. En tant qu'économiste préoccupé par les applications des théories, Hellet cherche une synthèse entre l'école autrichienne et l'école allemande pour des buts pratiques. Voir Aladár Madarász, « Heller Farkas (1877-1955) », Közgazdász, octobre 2000.

24. Le seul éminent économiste slovaque à trouver un poste au sein de la nouvelle université, peu après sa création, fut Milan Hodža.

25. Basch reste marqué par sa formation et, de ce fait, proche de la pensée autrichienne, même lorsqu'il est leur critique. Ainsi, en 1944, il publie une recension assez critique de l'article de Mises, Omnipotent Government, dans la revue American Economic Review. Engliš considère que Basch est le membre le plus important de l'école d'économie de Prague. Quand Basch émigre pour faire partie du gouvernement tchécoslovaque en exil pendant la seconde guerre mondiale, il occupe une position majeure et représente le gouvernement tchécoslovaque à la conférence de BrettonWoods de 1944. Il occupe ensuite un poste à l'université Columbia.

26. En 1926, Basch est remplacé à l'université Comenius par un autre économiste tchèque, Cyril Čechrák, institutionnaliste, proche des juristes et sceptique quant aux enseignements de l'école autrichienne. Les étudiants stimulés par Basch furent frustrés par cet enseignement jusqu'en 1938, l'année où Čechrák compte parmi les professeurs tchèques expulsés de Slovaquie. Pour une vue d'ensemble de la carrière et des contributions de Čechrák, voir Ilona Bažantová, «Cyril Čechrák ", dans Petra Skřejpková et al., Antologie československé právní vědy v meziválečném období (1918-1938) (Anthologie de science juridique tchécoslovaque dans l'entre-deux-guerres), Praha, Linde, 2009, p. 570-575.

27. Rudolf Briška, Národné hospodárstvo (Économie nationale), Bratislava, Družstevné vydavateL’stvo a kníhkupectvo v Bratislave, 1943.

28. Imrich Karvaš, Základy hospodárskej vedy [1947] (Fondements des sciences économiques), Bratislava, Veda, 1999.

29. «En 1898, l'Assemblée représentative de Carniole [nom que prend alors le territoire que nous appelons Slovénie] réclame la création d'une université avec des facultés de philosophie, de droit et de théologie. Un fonds est établi pour l'université et deux bourses sont financées, destinées à des Slovènes souhaitant accomplir leur agrégation à la faculté de droit ou de philosophie en Autriche, et prêts à accepter un poste de professeur à l'université de Ljubljana à leur retour » (Jožef Ciperle, Ustanovitev Univerze v Ljubljani v letu 1919: občasna razstava od decembra 2009 do februarja 2010 [Création de l'université de Ljubljana en 1919 : un exposé provisoire, décembre 2009-février 2010], V Ljubljani, Univerza, 2009, p. 87. L'influence de cette décision se voit rapidement dans le nombre d'étudiants slovènes au sein des universités autrichiennes : « En trente ans (1880-1910), le nombre des étudiants slovènes dans des universités ou écoles supérieures autrichiennes passe de quelque 350 (1880) à près de 1000 (1913)» (ibid., p. 7).

30. Toutefois, la proximité entre les langues tchèque et slovaque est également un facteur à prendre en compte à cet égard (N.D.L.R.)

31. Andrej Sušjan, «Ekonomski učbenik Valentina Žuna iz leta 1908: okoliščine nastanka ter nekatere vsebinske in jezikovne značilnosti » (Le manuel d'économie de Valentin Žun de 1908 : 
les circonstances de sa création et quelques caractéristiques relatives à son contenu et à son langage), Kronika: casopis za slovensko krajevno zgodovino (Chronique. Revue d'histoire locale slovène), $\mathrm{n}^{\circ}$ 61.1, 2012, p. 126.

32. Ivan Žmavc a étudié à Graz et à Prague. Il devint le tout premier professeur d'économie à Ljubljana en 1919. Il ne resta à Ljubljana que pour une brève période et il retrouva son poste précédent à Prague, à la bibliothèque universitaire. En conséquence, le premier professeur d'économie qui joua un rôle éminent à Ljubljana fut Milko Brezigar. Il a étudié à Vienne, est devenu journaliste et auteur actif en matière d'économie. Avant la première guerre mondiale, il écrivait en langue allemande sur des sujets variés. En 1918, son premier livre en slovène porte sur l'économie slovène: Osnutek slovenskega narodnega gospodarstva (Projet d'économie nationale slovène), Ljubljana, Založila in izdala «Omladina » - natisnila zvezna tiskarna, 1918. Il est recruté par l'université de Llubljana.

33. «À Ljubljana, malgré sa petite dimension et la marginalité de sa position (par rapport aux métropoles scientifiques et économiques qu'il connaissait), il [Bilimović] trouve un milieu suffisamment calme qui lui permet de se livrer à des travaux scientifiques. Il est en constante correspondance avec les meilleurs économistes allemands et autrichiens. Sa correspondance avec Friedrich von Hayek est particulièrement importante. Il publie beaucoup en allemand, ce qui s'explique par l'importance scientifique de Vienne, où la célèbre école autrichienne de pensée économique est créée à la fin du XIX siècle ", rapportent Alja Brglez et Matej Seljak dans Ruski Profesorji na Univerzi $v$ Ljubljani (Professeurs russes à l'université de Ljubljana), Ljubljana, Inštitut za Civizacijo in kulturo, 2007, p. 15. Voir aussi Hansjörg Klausinger, op. cit., p. 9-43. Nous tenons à remercier Blaz Remic d'avoir attiré notre attention sur ces points.

34. Voir Aleksandr Bilimovič, «Begründung der Teleologie als Form des empirischen Erkennens ", Zeitschrift für Nationalökonomie, 1931, p. 296-301; Aleksandr Bilimovič, "Meine Antwort an Herrn Prof. Dr. Karel Engliš », Zeitschrift für Nationalökonomie, vol. 4, n 4, 1933, p. 521-526; Karel Engliš, «Bilimovičovy námitky proti teleologické teorii národohospodářské » (Les objections de Bilimovič à la théorie téléologique de l'économie nationale), Obzor národohospodářský, n³7, 1932, p. 585-609; «Zum Problem der teleologischen Theorie der Wirtschaft », Zeitschrift Für Nationalökonomie Journal of Economics, vol. 4, n² 2, 1933, p. 220-242.

35. On se concentre dans cette partie sur les contributions tchèques à l'école autrichienne, cela ne signifie pas que d'autres nations d'Europe centrale n'ont pas contribué éminemment. En 2017, Marcin Chmielowski a réalisé un documentaire intitulé "Zapomniani » ("Ceux qui ont été oubliés ») sur les économistes de Cracovie pendant l'entre-deux-guerres. Il indique qu'ils étaient en un certain sens des "Autrichiens polonais ». S’il semble clair que les économistes de Cracovie se sont souvent inspirés des Autrichiens, la question de savoir comment les économistes de Cracovie ont contribué à l'école autrichienne doit encore faire l'objet d'une recherche plus approfondie. Emil Kauder a évalué les contributions de Čuhel, ainsi que celles de Bilimović, au développement du concept de mesure de l'utilité dans son histoire de l'utilité marginale et il les considère comme des contributions éminentes à l'école autrichienne. Voir Emil Kauder, A History of Marginal Utility Theory, Princeton, Princeton University Press, 1965, p. 197-199.

36. Voir Jaroslav Krameš, op. cit., p. 928.

37. Marek Hudík, « František Čuhel (1862-1914) », New Perspectives on Political Economy, vol. 3, nº 1, 2007, p. 3-14.

38. Slutsky cite Čuhel dans sa célèbre étude sur le budget des consommateurs, publiée pour la première fois en italien. Slutsky soutient que la loi de Gossen est une proposition empirique, et non une vérité rigoureusement prouvée, comme plusieurs chercheurs l'ont déjà fait valoir, et parmi lesquels il mentionne Edgeworth, Pantaleoni, Pareto, Marshall et Čuhel aussi. Eugen E. Slutsky, «Sulla teoria del bilancio del consumatore », Giornale degli Economisti e Rivista di Statistica, $\mathrm{n}^{\circ}$ 51, juillet 1915, traduit du russe par O. Ragusa, p. 1-26; trad. anglaise : « On the Theory of the 
Budget of the Consumer ", G. J. Stigler et K. J. Boulding (trad.), Readings in Price Theory (American Economic Association), Chicago, Richard D. Irwin Inc., 1952, p. 27-56.

39. Wesley C. Mitchell cite Čuhel dans son étude sur le comportement humain et les sciences économiques dans laquelle il examine les relations entre l'économie et la psychologie. Il y souligne que «Wicksteed, Chapman, Pareto, Schumpeter, Čuhel et Davenport disent tous que les préférences sont importantes et leurs origines sont sans importance.». Voir « Human Behavior and Economics: A Survey of Recent Literature ", Quarterly Journal of Economics, vol. 29, $\mathrm{n}^{\circ} 1$, novembre 1914.

40. Theo Surányi-Unger critique le concept de Čuhel du bien-être collectif et des acteurs collectifs. Voir Die Entwicklung der theoretischen Volkswirtschaftslehre im ersten Viertel des 20. Jahrhunderts, Jena, G. Fischer, 1927 ; traduit en anglais : Economics in the Twentieth Century [1932], Edwin Seligman (dir.), London, Routledge, 2003.

41. Fritz Machlup, « Rejoinder to a Reluctant Ultra-Empiricist », Southern Economic Journal, vol. 22, $\mathrm{n}^{\circ} 4$, avril 1956, p. 483-493.

42. Hoover Institution Archives, Hayek's files, box 104, folder 27.

43. «Bien qu'il contienne plusieurs suggestions intéressantes, il demeure manifestement insatisfaisant dans son ensemble. Pour la plupart, ce n'est qu'une déclaration de vérités bien connues avec une nouvelle terminologie barbare ", écrit Henry Raymond Mussey («Reviewed Work: Zur Lehre von den Bedürfnissen by Franz Cuhel », Political Science Quarterly, vol. 24, n 2, juin 1909, p. 324). Kirzner avait inclus l'ouvrage de Čuhel dans l'ensemble des 31 études majeures de la période allant de 1870 à 1970 rédigées au sein de la tradition autrichienne ; voir Israel M. Kirzner (éd.), Classics in Austrian Economics: A Sampling in the History of a Tradition, London, Pickering and Chatto, 1994, vol. 1. L'article de Čuhel figure dans le premier volume concernant les textes rédigés avant le déclenchement de la guerre mondiale (ceux de Menger, de Böhm Bawerk et de Wieser). Sur la question du rapport de l'école autrichienne à la psychologie, voir Gilles Campagnolo, «L'école autrichienne était-elle une école "psychologique” en économie aux yeux de Carl Menger? », infra.

44. Eugen Maria Schulak et Herbert Unterköfler, op. cit., p. 15 : «That subjective value is not measurable and therefore also not calculable was then first proven by Franz Cuhel. »

45. En 1947, Engliš devient recteur de l'université Charles de Prague.

46. «Cette école est détruite par le régime communiste : la plupart de ses représentants sont emprisonnés et persécutés sans aucune chance de reprendre leurs activités universitaires et leurs recherches ; leurs livres sont enlevés des bibliothèques et leur centre principal - la faculté de Droit de l'université de Brno - est fermée en 1950. » Voir Ilona Bažantová, «Czech Economist Karel Engliš and his Relation to the Austrian School in the First Half of the $20^{\text {th }}$ Century ", Prague Economic Papers, $\mathrm{n}^{\circ}$ 2, 2016, p. 234-246, p. 236.

47. Josef Šíma et Tomáš Nikodým, "Classical liberalism in the Czech Republic ", Econ Journal Watch, vol. 12, $\mathrm{n}^{\circ}$ 2, 2015, p. 274-292.

48. Israel Kirzner, The Economic Point of View, Menlo Park, Kirzner Institute for Humane Studies, 1976.

49. Le livre de Mises, publié en 1922, Le Socialisme, portait le sous-titre «Étude économique et sociologique " (Ludwig von Mises, Socialism: An Economic and Sociological Analysis, New Haven, Yale University Press, 1951). "Son essai suivant [un essai datant de 1928 qui est l'un des essais rassemblés en 1933 et publiés en un volume intitulé Grundprobleme der Nationalökonomie], paru sous le titre "Sociologie et histoire, enquête sur la science théorique de l'action humaine et de l'histoire" ». Mises allait explicitement indiquer bien plus tard le point suivant: "Là, j'avais d'abord commis l'erreur d'utiliser le terme "sociologie" pour désigner la théorie de l'action humaine. J'aurais dû utiliser le terme "praxéologie". » Voir Memoirs, Alabama, Ludwig von Mises Institute, 2009, p. 106. 
50. Mises se réfère au livre de Karel Engliš, Begründung der Teleologie als Form des empirischen Erkennens, Brünn, R. M. Rohrer, 1930.

\section{RÉSUMÉS}

Cette contribution analyse l'influence intellectuelle et sociale de la pensée économique autrichienne dans ses débuts en Europe centrale. Elle se concentre sur le transfert des concepts autrichiens à l'intérieur d'une communauté socio-économique d'Europe centrale alors naissante, dans un premier temps au sein d'un pays unique, c'est-à-dire dans l'Empire austro-hongrois avant la Première Guerre mondiale, puis, dans un second temps, au sein de pays indépendants au cours de la période de l'entre-deux-guerres. Aussi cet article offre-t-il un aperçu du transfert et de l'accueil des concepts de cette école de pensée dans les communautés économiques tchèque, polonaise, hongroise, slovène et slovaque. En outre, il s'agit d'étudier la contribution de savants non autrichiens (de par leur nationalité) à la pensée économique dite « autrichienne ».

Der Aufsatz analysiert den intellektuellen und sozialen Einfluss der Anfänge der Österreichischen ökonomischen Schule auf Zentraleuropa. Dabei konzentriert er sich auf den Transfer des Österreichischen Konzepts im Inneren der sozio-ökonomischen Gemeinschaft, welche damals im Entstehen war, zuerst im Inneren eines einzigen Landes, d.h. in der Österreichisch-Ungarischen Monarchie vor dem Ausbruch des Ersten Weltkriegs ; hierauf, in einer zweiten Periode, während der Zwischenkriegszeit, im Inneren der unabhängig gewordenen Länder. So bietet der Beitrag einen Überblick über den Transfer und die Aufnahme der Konzepte dieser Denkschule in den Wirtschaftsgemeinschaften von Tschechien, Polen, Ungarn, Slowenien und der Slowakei. Auf diese Weise werden außerdem die Beiträge von Wissenschaftlern, die aufgrund ihrer Nationalität nicht zu Österreich gehören, zum so genannten „österreichischen“ ökonomischen Gedankengut untersucht.

This paper discusses intellectual and social influence of the early Austrian economic thought in Central Europe. We focus on the transfer of early Austrian ideas into the economic community of Central Europeans: firstly, within one country, that is to say within the Austrian Empire before World War One, and secondly, across independent countries, during the Interwar period. Thus, we provide insights into the transfer and acceptance of these ideas by Czech, Polish, Hungarian, Slovenian and Slovak ethnic economic academic communities. We also discuss the contributions of ethnically non-Austrians to the development of Austrian economic thought.

\section{INDEX}

Schlüsselwörter : ideenaustausch, österreichische schule, nationalökonomie, Polen, Tschechoslowakei, Ungarn

Keywords : austrian school, economics, history of ideas, Czecho-Slovakia, Hungary, Poland

Mots-clés : école autrichienne, économie, histoire des idées, Hongrie, Pologne, Tchécoslovaquie 


\section{AUTEURS}

\section{JULIUS HORVÁTH}

Université d'Europe centrale / Central European University, Budapest

\section{TOMÁŠ KRIŠTOFÓRY}

Université J. E. Purkyně, Ústí nad Labem 